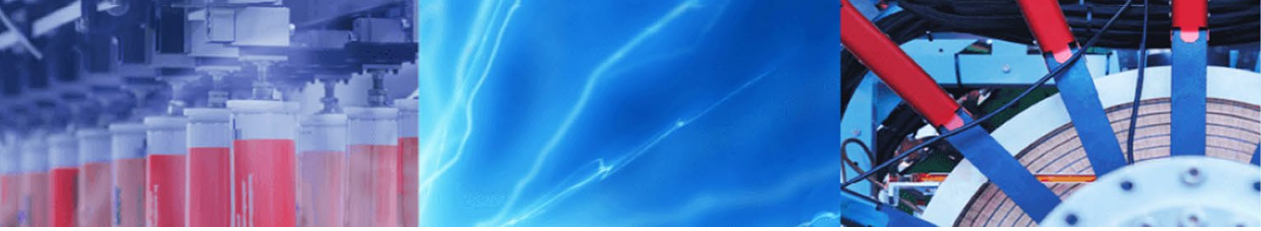

Research Article

\title{
Nature-inspired and hybrid optimization algorithms on interval Type-2 fuzzy controller for servo processes: a comparative performance study
}

\author{
Ritu Rani De (Maity) ${ }^{1}$ [D $\cdot$ Rajani K. Mudi ${ }^{1}$. Chanchal Dey ${ }^{2}$
}

Received: 23 March 2020 / Accepted: 9 June 2020 / Published online: 27 June 2020

(c) Springer Nature Switzerland AG 2020

\begin{abstract}
In this paper, performance evaluations of six well-known nature-inspired algorithms have been reported containing genetic algorithm, cuckoo search, particle swarm optimization, differential evolution, bee colony, and combined particle swarm optimization and differential evolution (CPSODE) algorithms. Based on these optimization algorithms, input and output scaling factors of an interval Type-2 fuzzy PID controller (IT2-FLC) are chosen for closed-loop servo tracking. Optimal values of the scaling factors are chosen by minimization of the objective function which is defined based on the closed-loop controller performance criteria. A detailed comparative analysis is reported based on the simulation and experimental results. Performance analysis reveals that improved performance, reliability, robustness, and lesser noise sensitivity are reported by IT2-FLC with the optimal values obtained by the hybrid algorithm CPSODE.
\end{abstract}

Keywords Genetic algorithm (GA) · Particle swarm optimization (PSO) · Differential evolution (DE) · Cuckoo search (CS) . Bee colony $(B C) \cdot$ Combined particle swarm optimization and differential evolution (CPSODE) algorithms · Interval Type-2 fuzzy controller · Servo tracking process

\section{Introduction}

Over the last decade, various nature-inspired algorithms have been reported to optimize a wide range of real-world engineering problems [1-5]. Considerable success of these algorithms remains in their capability to produce highquality solution within reasonable time. Problem solving through optimizations usually becomes complex with the size of optimization parameters involved, space complicity, length of execution time, and many other factors. Genetic algorithm (GA) [6, 7], particle swarm optimization (PSO) [8], differential evolution (DE) [9], bee colony (BC) [10], cuckoo search (CS) [11], and recently developed hybrid algorithm like combined PSO and DE (CPSODE) [12] are some of the most popular and very recent nature-inspired algorithms which are quite well accepted by the engineering community. In the reported study, these optimization algorithms have been exercised toward performance enhancement of a servo tracking process.

DC servo system positioning is widely used in various motion control applications for robotic manipulators [13, 14]. However, nonlinearity of a motor and uncertainty have significant role in influencing the closed-loop performance where load changes abruptly. Thus to achieve superior closed-loop response in such applications, interval Type-2 fuzzy logic controller [15-20] is considered to be an appropriate choice. Literature survey reveals that IT2-FLC [21-26] has been successfully implemented and found to be more competent than classical controllers in various applications $[13,14,24,25,27,28]$. To encompass

$\triangle$ Ritu Rani De (Maity), ritu_maity_8@yahoo.co.in; Rajani K. Mudi, rajanikanta.mudi@jadavpuruniversity.in; Chanchal Dey, cdaphy@ caluniv.ac.in | ${ }^{1}$ Department of Instrumentation and Electronics Engineering, Jadavpur University, Kolkata, India. ${ }^{2}$ Instrumentation Engineering, Department of Applied Physics, University of Calcutta, Kolkata, India. 
the uncertainties in a systematic manner, Zadeh [15] introduced the concept of fuzzy sets, and especially for Type-2 fuzzy sets, footprint of uncertainty (FOU) in membership function (MF) [18-20] has the ability for managing the vagueness and the uncertainty present in the process model $[18-20,22,24]$. This fact has been substantiated by a number of works reported in the literature where Type-2 fuzzy sets $[21-26,28,29]$ are found to be more capable to perform satisfactorily with higher degree of uncertainties compared to Type-1 fuzzy sets [27]. The only limitation remains with its computational complicity; however, it can be reduced considerably by choosing center-of-sets (COS)-type reduction method [20].

Here, well-known double-input-type fuzzy PID (FPID) [30-32] controller is considered where the choice of scaling factors (SFs) has an important role toward desired closed-loop performance. Usually, the values of the scaling factors are chosen by trial-and-error method. However, in the proposed work for proper designing of the IT2-FLC popular heuristic search algorithms like GA [6, 7], PSO [8], DE [9], BC [10], CS [11], and CPSODE [12] are exercised to obtain the optimal values of the input-output SFs. A comparative study has also been reported depicting the performance comparison among the different reported algorithms under load disturbance, model uncertainty, and measurement noise. Subsequent section, i.e., Section 2, briefly describes the prior mentioned optimization algorithms. Section 3 includes the details of the interval Type-2 fuzzy controller (IT2-FLC). Section 4 describes the experimental results related to servo tracking involving SRV02 [33] and QUBE-Servo-2 [34] along with the optimal scaling factors for the concerned IT2-FLC. A comparative study has also been made based on the simulation and experimental results, and finally, the conclusion is provided at the end.

\section{Nature-inspired optimization algorithms}

\subsection{Genetic algorithm (GA)}

GA is the most widely accepted optimization algorithm, primarily designed by Jon Holland [6]. GA [7] employs multi-directional search by initiating the process through a population of possible solutions, and it does not suffer from local optima as it has higher probability of escaping it. This algorithm starts with a group of chromosome known as population where the population size is related to the complexity of the problem. The initial choice of population is done randomly, and this algorithm employs three operations (selection, crossover, and mutation) on the population to reach toward the optimal solution.

'Selection' procedure includes the picking up of members of the mating pool from the current population with the help of proper selection procedure. The purpose of this action is to improve the solution over successive generations. 'Crossover' operation is helpful to share information among the chromosomes and propagate to the new generation of population as offspring of parent chromosome. Hence, the 'crossover' operation makes GA to enlarge the search space. The crossover probability $\rho_{c}$ is the probability of crossover that takes place between two parent chromosomes where $\rho_{\mathrm{c}}$ has some predefined value. If $\rho_{\mathrm{c}} \geq r$ (where $r$ is a random number, $r \in[0,1]$ ), then crossover occurs; otherwise, the parent chromosome is directly copied to the mating pool. For crossover, a crossover point is randomly generated integer in the range character length of the chromosome. The crossover operation consists of swapping the segments of the parent chromosomes from the one crossover point or multi-point crossover. Here, we have done crossover using swapping of the segments of parent chromosome. 'Mutation' operation imparts a small change at a random position within a chromosome. The intention of mutation in GA is to salvage from local optima and explore adequately every region of the search space. The mutation probability $\left(\rho_{\mathrm{m}}\right)$ is intentionally kept low; otherwise, it will disturb the search space too much and have difficulty in convergence.

\subsection{Particle swarm optimization (PSO)}

PSO technique is inspired by fish and bird swarm intelligence, and it was originally reported by Kennedy et al. [8] In PSO algorithm, an optimization problem under investigation is presented by the position of a particle vector. Each particle has a position as well as a current velocity, which represents the magnitude and direction toward achieving a better solution. In each iterative cycle, the fitness of each particle is evaluated based on an objective function $f(x)$. At every iteration, velocity $(v(t))$ for each particle is updated based on its current velocity and local as well as global swarm information as given by Eq. 1 a. Subsequently, new position for each particle is updated using Eq. 1b:

$v(t+1)=w \cdot v(t)+\left(c_{1} \cdot r_{1}(p(t)-x(t))\right)+\left(c_{2} \cdot r_{2}(g(t)-x(t))\right)$

$x(t+1)=x(t)+v(t+1)$ 
In Eq. 1a, present velocity is obtained based on the weighted velocity of the previous sampling instant. The other parameters of Eq. $1 \mathrm{a}$ are the local $(p(t))$ and global $(g(t))$ positional information of the particle. Here, $c_{1}$ and $c_{2}$ are the cognitive components related to the local and global weight, respectively, and both of them are considered to be two $\left(c_{1}=c_{2}=2\right)$. In Eq. $1 \mathrm{a}, r_{1}$ and $r_{2}$ are the random variables and their values are in the range $[0,1]$ and $w$ is the inertia weight whose value decreases from 0.9 to 0.4 with the number of iteration. Such technique of choosing inertia weight offers better performance compared to random choice of inertia weight. Based on some predefined criteria related to the convergence of fitness value defined as the reciprocal of the objective function $(f(x))$, optimal particle position vector is achieved after a number of iterations.

\subsection{Cuckoo Search (CS)}

Cuckoo Search (CS) is one of the most popular natureinspired metaheuristic algorithms, developed by Yang and Deb [11]. It incorporates Lévy flight method rather than simple isotropic random walks which makes CS potentially efficient than GA. As per the CS algorithm, cuckoo lays one egg at a time and dumps it in a random nest. Secondly, the best nests with high quality will be transferred to the next generation. Thirdly, the available host nest number remains fixed, and the eggs laid are discovered by the host bird with a probability $\left(\rho_{\mathrm{a}}\right)$ within 0 and 1 . In such case, the host bird can determine either to get rid of the egg or simply abandon the nest and build a new nest (with new random solutions). For minimization problem, the fitness function is reciprocal of the objective function.

The Lévy flight method used while generating a new solution for ith cuckoo is given by Eq. 2:

$x_{i}(t+1)=x_{i}(t)+\alpha \oplus$ Lé vy $(\lambda)$

Here, Lévy flight $(1<\lambda \leq 3)$ is a random walk phenomenon, while the random step length is drawn from a Lévy's distribution, which has an infinite variance with an infinite mean. For this reason, CS has the global convergence property stronger than PSO. As a result, PSO may converge prematurely to local optima, whereas CS may converge to global optima. In Eq. 2, $x_{i}$ is the chosen nest by the ith cuckoo and the value of step size $(a)$ is considered to be unity.

\subsection{Differential evolution (DE)}

DE is a stochastic, population-based optimization algorithm introduced by Storn and Price in 1996 [9]. This algorithm has been successfully used in different areas of science and engineering. The success and appreciation of DE algorithm crucially depend on appropriate selection of trial vector generation strategies and their control parameters. It mainly involves trial-and-error scheme for searching. In DE, three crucial control parameters are population size (NP), scaling factor (F), and crossover rate (CR). These parameters need to be properly tuned to successfully solve an optimization problem. This algorithm undergoes initialization of the population, subsequently mutation, followed by recombination. After recombination, it again goes through selection process and if the termination criterion is not reached, then same steps are repeated for the next generation with the selected candidates of the population.

DE algorithm involves a population NP with $d$-dimensional individual vectors, $X_{i, G}=\left(x_{i 1, G}+x_{i 2, G}+\cdots x_{i d, G}\right)$ where $i=1,2, \ldots, \mathrm{NP}$, from one generation to the next. $X_{i}$ is initialized, and then at each generation $\mathrm{G}$, each target vector employs mutation and forms mutant vector by their weighted difference

$v_{i G}=\left\{v_{i 1, G}, v_{i 2, G}, \ldots, v_{i N P, G}\right\}$ and

$V_{i G+1}=X_{i r 1, G}+F\left(X_{i r 2, G}-X_{i r 3, G}\right)$.

In Eq. 3a, $F$ is the scaling factor lies in between 0 and 1 . $X_{i 1 r, G}, X_{i 2 r, G}, X_{i 3 r, G}$ are randomly selected $r=1,2, \ldots$, NP. After mutation and crossover with each pair of mutation vector, the trial vector $U_{i G+1}$ for each individual $X_{i G+1}$ is formed:

$u_{i j, G+1}= \begin{cases}v_{i j, G+1} & \text { if } \operatorname{rand}_{j}(0,1) \leq \mathrm{CR} \text { or } j=j_{\text {rand }} \\ x_{i j, G+1} \text { otherwise }\end{cases}$

where $i=1,2 \ldots, \mathrm{NP}$ and $j=1,2, \ldots, d$. $j_{\text {rand }}$ is a random number $[1, d]$, and $C R$ is the crossover rate. Subsequently, to decide whether to select or not $U_{i, G+1}$ for the next generation fitness function $f\left(U_{i, G+1}\right)$ and $f\left(X_{i, G+1}\right)$ are compared:

$X_{i, G+1}= \begin{cases}U_{i, G} & \text { if } f\left(U_{i, G}\right)<f\left(X_{i, G}\right) \\ X_{i, G} & \text { otherwise }\end{cases}$

The success and appreciation of DE algorithm crucially depend on appropriate selection of trial vector generation and the control parameters.

\subsection{Bee colony (BC)}

The bee colony $(\mathrm{BC})$ algorithm is proposed by Karaboga in 2005 [10] which simulates the foraging behavior of bee colony. This algorithm is based on the characteristics observed in nature by bees' colony like it can extend itself over in multiple direction and over long distances (more than $10 \mathrm{~km}$ ) to avail a large number of food sources, and have the capacity of memorization, learning and 
transmission of information in colony, and thus forming the swarm intelligence.

In BC algorithm for the minimal model of swarm-intelligent forages selection in a honey bee colony, it consists of three kinds of bees: employed bees $\left(n_{\mathrm{e}}\right)$, onlooker bees $\left(n_{\mathrm{o}}\right)$, and scout bees $\left(n_{\mathrm{s}}\right)$. Half of the colony consists of employed bees, and the other half includes onlooker bees. The responsibility of the employed bees is to exploit the nectar sources explored before and convey information to the waiting onlooker bees in the hive about the quality of the food source sites which they are exploiting. Based on the shared information of the employed bee, the onlooker bees wait in the hive and decide on a food source to exploit. If the source is abandoned, she becomes a scout and starts to randomly search the environment in order to find a new food source.

\subsection{Hybrid algorithm CPSODE}

To minimize the limitations involved with PSO [8] such as trapping at local minima and premature convergence, PSO is combined with DE [9] and it results CPSODE [12]. This algorithm has a novel feature which incorporates cooperation mechanism into the evolution of search agents. To obtain the minima, total population is divided into two groups. One group employs PSO evolution strategy, and the other adopts DE algorithm. In this algorithm, each particle is evolved based on cooperation information sharing of other particles. A certain threshold of trapped particles is there, i.e., if threshold $S$ is reached, the trapped particles will be replaced by new particles from the other evolution group.

In CPSODE, the PSO group is denoted by $G_{\mathrm{PSO}}$ and the $D E$ group by $G_{D E}$. Each particle position can be denoted as $M$-dimensional search space with $P$ number of particles in $G_{\mathrm{PSO}}$ and $D$ number of particles in $G_{\mathrm{DE}}$. Initialization of each particle by individual DE and PSO strategy are done. Next, ith particle position can be represented as for PSO group, $P_{i}=\left(p_{i 1}, p_{i 2}, \ldots \ldots p_{i m}\right), \quad i=1,2 \ldots, P$. Each particle contains its previous best position, $B_{i}=\left(b_{i 1}, b, \ldots b_{i m}\right)$, and velocity, $v_{i}=\left(v_{i 1}, v, \ldots v_{i m}\right), i=1,2 \ldots, P . B_{\mathrm{g}}$ represents the best solution over the whole population. After each iteration step, the particle updates by velocity and position by Eqs. $4 \mathrm{a}$ and $4 \mathrm{~b}$ :

$V_{i m}(t+1)=w V_{\mathrm{m}}(t)+c_{1} \times r_{1} \times\left(B_{i m}-P_{i m}\right)+c_{2} \times r_{2} \times\left(B_{g m}-P_{i m}\right)$

$P_{i m}(t+1)=P_{i m}(t)+V_{i m}(t+1)$

where $B_{i m}$ is the $m$ th vector for the best position of particle $i$ and $B_{g m}$ is the $m$ th vector for the best position of the current population. Here, $r_{1}, r_{2} \in[0,1]$ are uniformly generated random number, and $c_{1}, c_{2}$ are the acceleration coefficients.

After certain number of iterations, some stagnant individuals in the population $G_{\mathrm{PSO}}$ and $G_{\mathrm{DE}}$ can be created. They are counted by

$\operatorname{Cnt}(t+1)=\left\{\begin{array}{l}1 \text { if }\left(f\left(X_{i}^{t}\right) \leq f\left(X_{i}^{t-1}\right) \leq f\left(X_{i}^{t-2}\right)\right) \\ 0 \text { otherwise }\end{array}\right.$,

The number of trapped particles in $G_{\mathrm{PSO}}$ and $G_{\mathrm{DE}}$ can be calculated by

$\mathrm{Cnt}_{\mathrm{PSO}}=\sum_{i=1}^{P} \mathrm{Cnt}_{i}, i \in G_{\mathrm{PSO}} ; \mathrm{Cnt}_{\mathrm{DE}}=\sum_{j=1}^{P} \mathrm{Cnt}_{j}, j \in G_{\mathrm{DE}}$

When $\mathrm{Cnt}_{\mathrm{PSO}}$ or $\mathrm{Cnt}_{\mathrm{DE}}$ reaches stopping criteria $\mathrm{S}$, then the trapped particles will be replaced by the filial generation of two particles from the other group. The mutation strategy is similar to DE. The new particle for the trapped particle becomes:

$P_{i}^{t+1}=P_{\min }+\operatorname{rand}(0,1) \times\left(P_{\max }-P_{\min }\right)$

where $\left(P_{\max }, P_{\min }\right)$ denotes the range of the other population. The counter of particles $i$ will be reset to zero, $\mathrm{Cnt}_{i}(t+1)=0$.

Here, CPSODE algorithm is utilized for proper designing of the Mamdani-type interval Type-2 fuzzy logic controller designing for servo tracking applications. Here, the optimized values for the scaling factors are searched out for designing of a Type-2 fuzzy controller which is capable to provide superior closed-loop performance.

\section{Interval Type-2 Fuzzy Logic controller}

The concept of Type-2 fuzzy set was introduced by Zadeh [15] in 1975. Further discussions of Type-2 fuzzy sets are elaborated in [18-20]. Literature survey reveals that most of the reported works $[29,30]$ related to Type-2 fuzzy logic controller designing involve Type-2 fuzzy sets where all points in the FOU have unity secondary membership grade. The use of interval Type-2 fuzzy set reduces computational complexity which is the prominent drawback of Type-2 FLC compared to Type-1 FLC [27]. Here, the designing optimal interval Type- 2 FLC is shown in Fig. 1 where the crisp inputs from the input sensors are first fuzzified into input MFs which are Type-2 fuzzy sets. Inference engine with the help of rule base and data base produces output MFs which are also Type-2 fuzzy sets. Interval Type-2 $\mathrm{FLC}$ has the similar inference engine and rule base as with Type-1, only the input and output MFs are of interval Type- 2 fuzzy sets. The inference engine combines the fired rules, and it maps the input Type- 2 sets to output Type- 2 


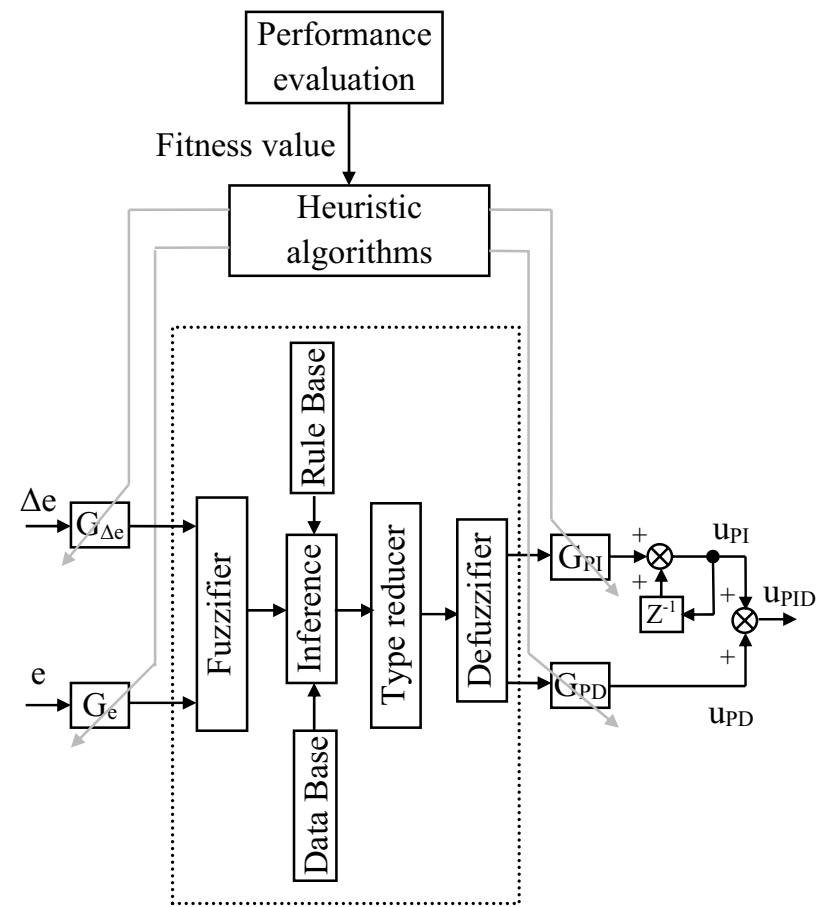

Type-2 FLC

Fig. 1 Block diagram of T2-FPID

fuzzy sets. Type reducer is used to convert the Type-2 fuzzy output sets into Type-1 fuzzy sets. Popular type reduction methods are KM method [18, 20], COS method [20], and uncertainty bound method [18]. After the type reduction, Type-1 output fuzzy sets are defuzzified [20] and the crisp output is obtained $[18,19]$.

In the literature, different types of Type-2 fuzzy controllers [30-32] are reported. Here, a simple but effective fuzzy $\mathrm{PI}+$ fuzzy PD-type controller structure is considered with a two-dimensional linear rule base and Mamdani-type inferencing [31] as shown in Fig. 1. For simplicity and ease of implementation, triangular MFs for error (e), change of error $(\Delta e)$, and $U$ (control action) with five fuzzy sets are employed as shown in Fig. 2. Observe that Fig. 2 shows

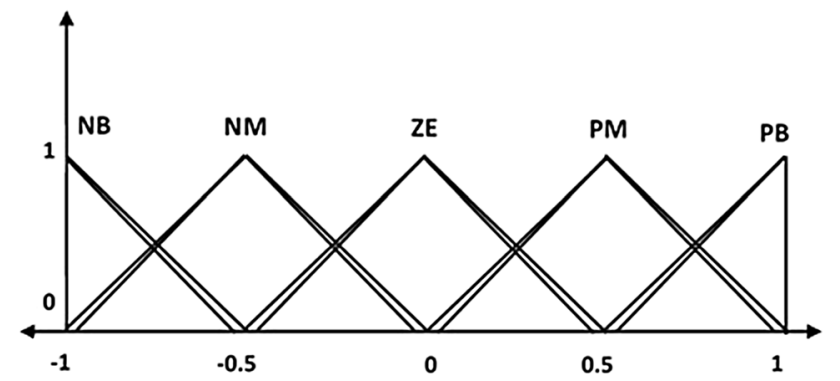

Fig. 2 Membership function of Type-2 fuzzy sets for $e, \Delta e, u, \Delta u$ fuzzy partitions for the same universe of discourse of input $(e, \Delta e)$ and output $(u)$ linguistic variables. The controller output $u$ is determined by rules of the form: If $e$ is $E$ and $\Delta e$ is $\Delta E$, then $u$ is $U$,

where $e(k)=r-y(k)$

$\Delta e(k)=e(k)-e(k-1)$

The input variables (e) and $\Delta(e)$ are partitioned by $N$ number of membership functions, and assuming that for each possible combination of the $N$ sets, there is a rule for this two domain inputs $\bar{X}_{1}, \breve{X}_{2}$, and the rule base comprises $N^{2}$ rules. The change in controller output $u$ is determined by the $n$th rule:

If $e$ is $\widetilde{X}_{1}$ and $\Delta e$ is $\widetilde{X}_{2}^{j}$, then $u^{n}$ is $U^{n}(i, j=1,2 \ldots N)$ and $U^{n}$ the distinct Type-2 fuzzy set and $\left[\underline{u}^{n}, \bar{u}^{n}\right]$ an intervall. Nyext, we compute the membership of input variable $\bar{X}_{1}, \bar{X}_{2}$ as $\left[\underline{\mu}_{\bar{X}}^{i}\right.$ (e), $\left.\bar{\mu}_{\bar{x}_{1}^{i}}(e)\right]$ and $\left[\underline{\mu}_{\bar{x}^{j}}(\Delta e), \bar{\mu}_{\bar{x}_{2}^{j}}(\Delta e)\right]$ for $i, j=1,2 \ldots N . \underline{\mu}_{\bar{x}_{1}{ }^{i}}(e)$ and $\bar{\mu}_{\bar{x}_{1}}(e)$ are the upper and lower membership grades of the interval firing set $\mu_{\bar{x}_{1}}(e)$. Similarly, $\underline{\mu}_{\bar{x}_{2}^{j}}(\Delta e)$ and $\bar{\mu}_{\bar{x}_{2}^{j}}(\Delta e)$ are the lower and upper membership grades of input variable $\mu_{\bar{X}_{2}^{j}}(e)$.

Next, we compute the firing interval of the $n$th rule:

$F^{n}(e, \Delta e)=\left[\underline{f}^{n}(e, \Delta e), \bar{f}^{n}(e, \Delta e)\right]$, where $n=1, \ldots N^{2}$.

$=\left[\underline{\mu}_{\bar{x}_{1}^{i}}(\mathrm{e}) \times \underline{\mu}_{\bar{x}_{2}^{j}}(\Delta e), \bar{\mu}_{\bar{x}_{1}^{i}}(e) \times \bar{\mu}_{\bar{x}_{2}}(\Delta e)\right]$,

$i=1,2 \ldots N$ and $j=1,2 \ldots N$

With the $F^{n}(e, \Delta e)$ and the corresponding rule consequents, we perform the center-of-sets-type reduction (COS):

$U_{\cos (e, \Delta e)}=\bigcup_{\substack{f^{n} \in F^{n}(e, \Delta e) \\ u^{n} \in U^{n}}} \frac{\sum_{n=1}^{N} f^{n} u^{n}}{\sum_{n=1}^{N} f^{n}}=\left[u_{l}, u_{r}\right]$

$u_{l}=\frac{\sum_{n=1}^{L} \underline{u}^{n} \bar{f}^{n}+\sum_{n=L+1}^{N} \underline{u}^{n} \underline{f}^{n}}{\sum_{n=1}^{L} \bar{f}^{n}+\sum_{n=L+1}^{N} \underline{f}^{n}} ; u_{r}=\frac{\sum_{n=1}^{R} \bar{u}^{n} \underline{\underline{f}}^{n}+\sum_{n=R+1}^{N} \bar{u}^{n} \bar{f}^{n}}{\sum_{n=1}^{R} \underline{f}^{n}+\sum_{n=R+1}^{N} \bar{f}^{n}}$

The switching points $L$ and $R$ are determined by Karnik and Mendel (KM) [20] algorithm such that $\underline{u}^{L} \leq u_{l} \leq \underline{u}^{L+1}$ and $\bar{u}^{R} \leq u_{r} \leq \bar{u}^{R+1}$. Finally, computation of the defuzzified output is 
Table 1 Fuzzy control rule base

\begin{tabular}{llllll}
\hline$\Delta e / e$ & $\mathrm{NB}$ & $\mathrm{NM}$ & $\mathrm{ZE}$ & $\mathrm{PM}$ & $\mathrm{PB}$ \\
\hline $\mathrm{NB}$ & $\mathrm{NB}$ & $\mathrm{NB}$ & $\mathrm{NB}$ & $\mathrm{NM}$ & $\mathrm{ZE}$ \\
$\mathrm{NM}$ & $\mathrm{NB}$ & $\mathrm{NB}$ & $\mathrm{NM}$ & $\mathrm{ZE}$ & $\mathrm{PM}$ \\
$\mathrm{ZE}$ & $\mathrm{NB}$ & $\mathrm{NM}$ & $\mathrm{ZE}$ & $\mathrm{PM}$ & $\mathrm{PB}$ \\
$\mathrm{PM}$ & $\mathrm{NM}$ & $\mathrm{ZE}$ & $\mathrm{PM}$ & $\mathrm{PB}$ & $\mathrm{PB}$ \\
$\mathrm{PB}$ & $\mathrm{ZE}$ & $\mathrm{PM}$ & $\mathrm{PB}$ & $\mathrm{PB}$ & $\mathrm{PB}$ \\
\hline
\end{tabular}

$U=\frac{u_{1}+u_{r}}{2}$

The rule base consisting of 25 rules for computing $u$ is shown in Table 1, which is a commonly used rule base designed in sliding mode principle [35]. From Fig. 1, we find that the final controller output $U$ is obtained by the relation:

$U=G_{\mathrm{PD}} \cdot u+G_{\mathrm{PI}} \cdot \Delta u$

In this IT2-FLC of fuzzy PID [30, 31] type, the values of the actual inputs $e$ and $\Delta e$ are mapped to the interval $[-1$, $1]$ by the input SFs, $G_{e}$, and $G_{\Delta e^{\prime}}$ respectively. The defuzzified output $u_{N}$ is translated into the actual output $u$ by the output $S F, G_{P 1}$, and $G_{P D}$. Initial settings with suitable values of $G_{e}, G_{\Delta e^{\prime}} G_{\mathrm{Pl}}$, and $G_{\mathrm{PD}}$ are found based on the knowledge about the process to be controlled and sometimes through trial. Here in this paper, we have used some heuristic algorithms to find the optimal values of the two input scaling factors for error $G_{e}$, change of error $G_{\Delta e}$ and two output scaling factor $G_{\mathrm{Pl}}$, and $G_{\mathrm{PD}}$.

\section{Results and settings}

In order to understand the effect of optimization on the scaling factors of the interval Type-2 fuzzy PID controller, both simulation and real-time experimentation have been done. The improvement of the IT2-FLC without optimization and with optimization is demonstrated in terms of different performance indices-percentage overshoot (\% O.S.), rise time $\left(t_{\mathrm{r}}\right)$, settling time $\left(t_{\mathrm{s}}\right)$, integral absolute error $(\mathrm{IAE})$, integral time absolute error (ITAE). Noise sensitivity of the IT2-FLC and optimal IT2-FLC is also evaluated in the presence of measurement noise.

\subsection{Optimization of parameters}

In this paper for the optimal value selection of the four parameters $G_{e}, G_{\Delta e}, G_{\mathrm{Pl}}$, and $G_{\mathrm{PD}}$ of the interval Type-2 fuzzy controller by the above-mentioned optimization algorithms CS, DE, PSO, GA, CPSODE, and BCA [6-12], a minimum value of the objective function is required. To achieve an optimal solution for set-point tracking and load
Table 2 Tuning values of the parameters for PSO, GA, CS, DE, and BCA

\begin{tabular}{|c|c|c|}
\hline Algorithm & Parameter & Value \\
\hline PSO & $\begin{array}{l}c_{1}, c_{2}, \text { population, Max. } \\
\text { iteration }\end{array}$ & $2,2,50,100$ \\
\hline GA & $\begin{array}{l}\rho_{\mathrm{c}^{\prime}} \rho_{\mathrm{m}} \text {, selection operator, } \\
\text { total chromosomes, } \\
\text { Max. iteration }\end{array}$ & $6.667,0.2,50,100$ \\
\hline CS & $\rho_{\mathrm{a}}$, nest, Max. iteration & $0.25,50,100$ \\
\hline DE & $\begin{array}{l}\mathrm{F}, \mathrm{CR} \text {, population, Max } \\
\text { iteration }\end{array}$ & $0.45,0.5,50,100$ \\
\hline BCA & Colony size, $n_{\mathrm{e}}, n_{\mathrm{o}}, n_{\mathrm{s}}$, limit & $\begin{array}{l}50,50 \% \text { of the colony, } 50 \% \\
\text { of the colony, } 1,2 \text {. }\end{array}$ \\
\hline CPSODE & $\begin{array}{l}c_{1}, c_{2}, w_{\min }, w_{\text {max }}, F \\
C R, \text { population, Max. } \\
\text { iteration }\end{array}$ & $\begin{array}{l}2,2,0.4,0.9,0.8,0.5,40 \\
\quad 100\end{array}$ \\
\hline
\end{tabular}

rejection phases, the following objective function $f(x)$ is considered involving absolute value of overshoot (\%OS), settling time $\left(t_{\mathrm{s}}\right)$, and integral absolute error (IAE):

Objective function $=a b s(\% \mathrm{OS})+t_{\mathrm{s}}+\mathrm{IAE}$

Each optimization algorithm is carried for 100 iterations and executed for 20 runs by proper setting of the parameters which are given in Table 2.

The optimization algorithms have been used to achieve the minimum value of the objective function which is based on the closed-loop performance using the Type-2 fuzzy controller in servo system. In this paper, using the above-mentioned optimization algorithms proper selection of the four parameters $G_{e}, G_{\Delta e}, G_{\mathrm{Pl}}$, and $G_{\mathrm{PD}}$, for the interval Type-2 fuzzy controller in the servo system: (i) nominal model and two experimental setup Quanser, Canada made (ii) SRV02, and (iii) QUBE-Servo-2, has been successively done. The optimal results of PSO, GA, BCA, CPSODE, DE, and CS for the different systems are given in Tables 3, 4, and 5. The servo system descriptions on which the experiments are performed are discussed in the following section.

\subsection{System description}

Quanser SRV02 In this section, we present the Quanser, Canada made SRV02 [33] rotary servo plant, pictured in Fig. 3 consisting of a DC motor enclosed in a solid aluminum frame and equipped with a planetary gear box. The motor has its own internal gearbox that drives external gears. The potentiometer and encoders measure the angular position of the load gear, and the tachometer measures its velocity. The schematic diagram of SRV02 is shown in Fig. 3. The electrical equation for the SRV02 servo system can be given by Eq. (13) as follows: 
Table 3 Optimization results of parameters $G_{\mathrm{e}}, G_{\Delta \mathrm{e}}, G_{\mathrm{Pl}}$, and $G_{\mathrm{PD}}$ for model in (42)
Table 4 Optimization results of parameters $G_{e^{\prime}} G_{\Delta e^{\prime}} G_{\mathrm{P},}$, and $G_{\mathrm{PD}}$ for SRV02 system
Table 5 Optimization results of parameters $G_{e^{\prime}} G_{\Delta e^{\prime}} G_{\mathrm{Pl}}$, and $G_{\mathrm{PD}}$ for QUBE-Servo-2

\begin{tabular}{lllllcc}
\hline Algorithm used & $G_{e}$ & $G_{\Delta e}$ & $G_{\mathrm{PI}}$ & $G_{\mathrm{PD}}$ & Fitness value & Execution time $(\mathrm{s})$ \\
\hline CS & 0.5168 & 27.9218 & 0.0228 & 0.12967 & 18.9036 & 70.849 \\
DE & 1.4812 & 47.2963 & 0.0998 & 1.00 & 9.1783 & 367.972 \\
GA & 0.7000 & 36.0000 & 0.0160 & 0.1000 & 25.3465 & 340.4465 \\
PSO & 0.500 & 20.0000 & 0.03075 & 0.0100 & 11.7096 & 185.7556 \\
BCA & 1.2779 & 31.7102 & 0.08306 & 0.4859 & 14.3742 & 173.974 \\
CPSODE & 0.500 & 37.0737 & 0.0100 & 0.1000 & 98.0392 & 780.976
\end{tabular}

\begin{tabular}{llllllc}
\hline Algorithm used & $G_{e}$ & $G_{\Delta e}$ & $G_{\mathrm{PI}}$ & $G_{\mathrm{PD}}$ & Fitness value & Execution time (s) \\
\hline CS & 0.74091 & 41.4513 & 0.08705 & 0.3533 & 0.6281 & 455.261 \\
DE & 0.51765 & 48.4522 & 0.06546 & 0.3357 & 0.5177 & 1495.980 \\
GA & 0.9000 & 50.0000 & 0.06400 & 0.6400 & 0.7572 & 2421.351 \\
PSO & 0.9715 & 50.0000 & 0.06431 & 0.3030 & 0.4890 & 1566.669 \\
BCA & 1.2815 & 45.4603 & 0.0506 & 0.64479 & 0.5683 & 12890.162 \\
\hline
\end{tabular}

\begin{tabular}{llllllc}
\hline Algorithm used & $G_{e}$ & $G_{\Delta \mathrm{e}}$ & $G_{\mathrm{PI}}$ & $G_{\mathrm{PD}}$ & Fitness value & Execution time(s) \\
\hline CS & 0.57145 & 27.2746 & 0.01483 & 0.4473 & 0.3761 & 147.9788 \\
DE & 0.7541 & 35.4677 & 0.0100 & 0.8497 & 0.4390 & 381.5669 \\
GA & 0.8333 & 44.0000 & 0.0820 & 0.7000 & 0.4141 & 1104.08 \\
PSO & 0.9054 & 46.7083 & 0.1000 & 0.0100 & 0.45513 & 286.9098 \\
BCA & 0.443 & 49.4319 & 0.0160 & 0.2757 & 0.46795 & 677.1070 \\
\hline
\end{tabular}

$e_{\mathrm{b}}(t)=K_{\mathrm{m}} w_{\mathrm{m}}(t)$

Here, $e_{\mathrm{b}}$ is the back-emf (electromotive) voltage, $w_{\mathrm{m}}$ the speed of the motor shaft and $K_{\mathrm{m}}$ the back-emf constant of the motor. Then by using KVL, we can write

$V_{\mathrm{m}}(t)-R_{\mathrm{m}} I_{\mathrm{m}}-L_{\mathrm{m}} \frac{\mathrm{d} I_{\mathrm{m}}(t)}{\mathrm{d} t}-K_{\mathrm{m}} w_{\mathrm{m}}(t)=0$

$I_{\mathrm{m}}(t)=\frac{V_{\mathrm{m}}(t)-K_{\mathrm{m}} W_{\mathrm{m}}}{R_{\mathrm{m}}}$

The mechanical equation of the system can also be derived. Equation of motion describing the speed of the load shaft is $w_{\mathrm{L}}$ and $\tau_{\mathrm{m}}$ the applied motor torque. Since SRV02 is a one-degree-of-freedom rotary system, Newton's second law of motion can be written as:

$J \cdot \alpha=\tau$

where $J$ is the moment of inertia of the body (about its center mass), $\alpha$ the angular acceleration of the system, and $\tau$ sum of the torques being applied to the body. According to Fig. 3, the SRV02 gear train along with the viscous friction acting are the motor shaft $B_{\mathrm{m}}$ and the load shaft $B_{1}$. Then, the load equation is as follows:

$J_{1} \frac{\mathrm{d} w_{\mathrm{L}}(t)}{\mathrm{d} t}+B_{1} w_{1}(t)=\tau_{1}(t)$

where $J_{l}$ is the moment of inertia of the load and $\tau_{1}$ the total torque applied on the load.

Again, the motor shaft equation is expressed as:

$J_{\mathrm{m}} \frac{\mathrm{d} w_{\mathrm{m}}(t)}{\mathrm{d} t}+B_{\mathrm{m}} w_{\mathrm{m}}(t)+\tau_{\mathrm{ml}}(t)=\tau_{\mathrm{m}}(t)$

where $J_{\mathrm{m}}$ is the motor shaft moment of inertia, and $\tau_{\mathrm{ml}}$ is the resulting torque acting on the motor shaft from the load torque.

Thus, the resulting torque at the load shaft from an applied motor torque can be written as:

$\tau_{l}(t)=n_{\mathrm{g}} K_{\mathrm{g}} \tau_{\mathrm{ml}}(t)$

$K_{\mathrm{g}}$ is the gear ratio, and $n_{\mathrm{g}}$ is the gearbox efficiency.

The planetary gearbox is directly mounted on the SRV02 motor and is represented by $N_{1}$ and $N_{2}$ gears, and the gear ratio is: 
Fig. 3 SRV02 DC motor armature circuit and gear train

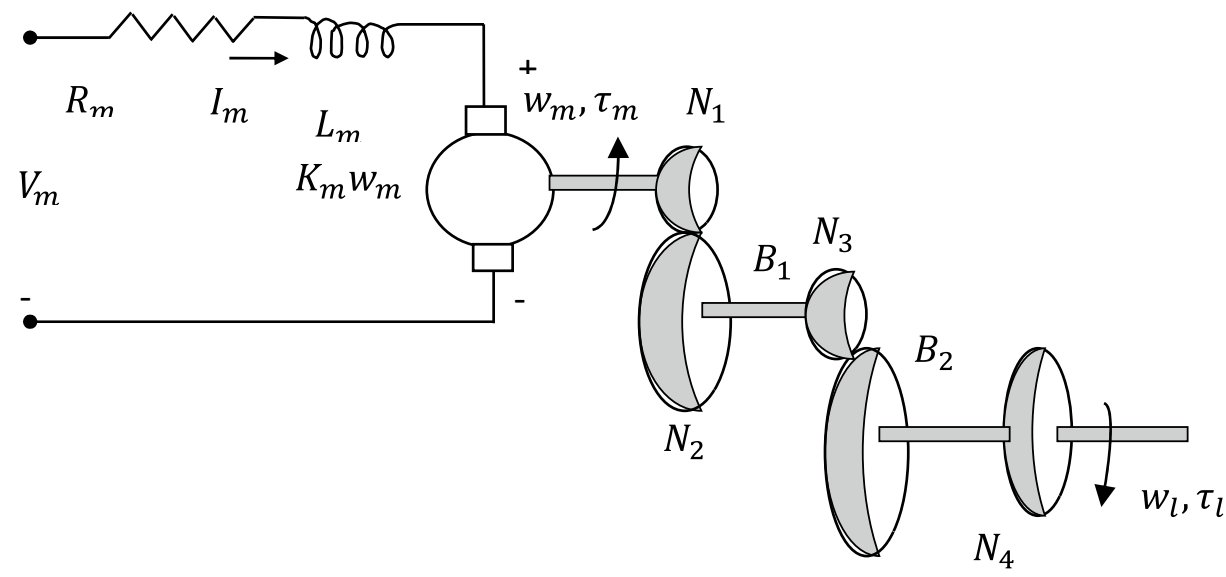

$K_{\mathrm{gi}}=\frac{N_{2}}{N_{1}}$

$\left(n_{\mathrm{g}} J_{\mathrm{m}} K_{\mathrm{g}}^{2}+J_{l}\right) \frac{\mathrm{d} w_{l}(t)}{\mathrm{d} t}+\left(n_{\mathrm{g}} B_{\mathrm{m}} K_{\mathrm{g}}^{2}+B_{l}\right) w_{l}(t)=n_{\mathrm{g}} K_{\mathrm{g}} \tau_{\mathrm{m}}(t)$

Next is the internal gear ratio. The motor gear $N_{3}$ and the load gear $\mathrm{N}_{4}$ are directly meshed together and are visible from outside as

$K_{\mathrm{ge}}=\frac{N_{4}}{N_{3}}$

The gear ratio of the SRV02 gear train is given by:

$K_{\mathrm{g}}=K_{\mathrm{ge}} K_{\mathrm{gi}}$

Thus, the torque seen at the motor shaft is:

$\tau_{\mathrm{ml}}(t)=\frac{\tau_{\mathrm{l}}(t)}{n_{\mathrm{g}} K_{\mathrm{g}}}$

where $n_{\mathrm{g}}$ is the gearbox efficiency and $K_{E}$ the gear ratio. Intuitively, the motor shaft must rotate $K_{\mathrm{g}}$ times for the output shaft to rotate one revolution:

$\theta_{\mathrm{m}}(t)=K_{\mathrm{g}} \theta_{\mathrm{l}}(t)$

The relationship between the angular speed of the motor shaft $w_{m}(t)$ and the angular speed of the load shaft $w_{1}(t)$ by taking time derivative is:

$w_{\mathrm{m}}(t)=K_{\mathrm{g}} w_{\mathrm{l}}(t)$

Resultant differential equation that describes the motion of the load shaft with respect to an applied motor torque is given below:

$J_{\mathrm{m}} K_{E} \frac{\mathrm{d} w_{\mathrm{l}}(t)}{\mathrm{d} t}+B_{\mathrm{m}} K_{\mathrm{g}} w_{\mathrm{l}}(t)+\frac{J_{\mathrm{l}} \frac{\mathrm{d} w_{\mathrm{L}}(t)}{\mathrm{d} t}+B_{\mathrm{l}} w_{\mathrm{l}}(t)}{n_{\mathrm{g}} K_{\mathrm{g}}}=\tau_{\mathrm{m}}(t)$

Collecting the coefficients in terms of the load, shaft velocity, and acceleration gives:
Defining the following terms:

$J_{\text {eq }}=n_{\mathrm{g}} J_{\mathrm{m}} K_{\mathrm{g}}^{2}+J_{l}, B_{\text {eq }}=n_{\mathrm{g}} B_{\mathrm{m}} K_{\mathrm{g}}^{2}+B_{l}$, Eq. (22) reduces to

$J_{\text {eq }} \frac{\mathrm{d} w_{l}(t)}{\mathrm{d} t}+B_{\text {eq }} w_{l}(t)=n_{\mathrm{g}} K_{\mathrm{g}} \tau_{\mathrm{m}}(t)$

Combining the electrical and mechanical equation, an expression has been derived combining the effect of load shaft speed in terms of the applied motor voltage. The motor torque is proportional to the following:

$\tau_{\mathrm{m}}(t)=n_{\mathrm{m}} k_{\mathrm{t}} l_{\mathrm{m}}(t)$

where $k_{\mathrm{t}}$ is the current-torque constant, $n_{\mathrm{m}}$ the motor efficiency, and $I_{\mathrm{m}}$ the armature current. We can express the motor torque with respect to the input voltage, $V_{m}(t)$, and load shaft speed $w_{m}(t)$ by substituting the motor armature current:

$\tau_{\mathrm{m}}(t)=n_{\mathrm{m}} k_{\mathrm{t}} \frac{\left(V_{\mathrm{m}}(t)-K_{\mathrm{m}} w_{\mathrm{m}}\right)}{R_{\mathrm{m}}}$

Again, we can express the above equation in terms of $V_{m}$ and $w_{l}$ by inserting the motor load-shaft speed equation:

$\tau_{\mathrm{m}}(t)=n_{\mathrm{m}} k_{\mathrm{t}} \frac{\left(V_{\mathrm{m}}(t)-K_{\mathrm{m}} K_{\mathrm{g}} w_{l}(t)\right)}{R_{\mathrm{m}}}$

Substituting Eq. (31) in Eq. (28), we get

$J_{\text {eq }} \frac{\mathrm{d} w_{l}(t)}{\mathrm{d} t}+B_{\text {eq }} w_{l}(t)=n_{\mathrm{g}} K_{\mathrm{g}} n_{\mathrm{m}} k_{\mathrm{t}} \frac{\left(V_{\mathrm{m}}(t)-K_{\mathrm{m}} K_{\mathrm{g}} w_{l}(t)\right)}{R_{\mathrm{m}}}$

$J_{\text {eq }} \frac{\mathrm{d} w_{l}(t)}{E t}+\left(B_{\text {eq }}+\frac{n_{\mathrm{g}} K_{\mathrm{g}}^{2} n_{\mathrm{m}} k_{\mathrm{t}}}{R_{\mathrm{m}}}\right) w_{l}(t)=\frac{n_{\mathrm{g}} K_{\mathrm{g}} n_{\mathrm{m}} k_{\mathrm{t}} V_{\mathrm{m}}(t)}{R_{\mathrm{m}}}$ 
$J_{\text {eq }} \frac{\mathrm{d} w_{l}(t)}{\mathrm{d} t}+B_{\mathrm{eq}, v} w_{l}(t)=A_{\mathrm{m}} V_{\mathrm{m}}(t)$

where $B_{\text {eq, } v}=B_{\text {eq }}+\frac{n_{\mathrm{g}} K_{\mathrm{g}}^{2} n_{\mathrm{m}} k_{\mathrm{t}}}{R_{\mathrm{m}}}$ and $A_{\mathrm{m}}=\frac{n_{\mathrm{g}} K_{\mathrm{g}} n_{\mathrm{m}} k_{\mathrm{t}}}{R_{\mathrm{m}}}$. The final derived Eq. (32) is a first-order system.

Here, $R_{\mathrm{m}}=2.6 \Omega$, the motor armature resistance; $n_{\mathrm{g}}=0.9$, the gear box efficiency; $K_{\mathrm{g}}=70$, the high-gear total gear ratio; $n_{\mathrm{m}}=0.69$, the motor efficiency; $k_{\mathrm{t}}=7.68 \times 10^{-3} \mathrm{~N}-\mathrm{m} / \mathrm{A}$, the motor current-torque constant; and $B_{\text {eq }}=0.015 \mathrm{Nm}(\mathrm{rad} / \mathrm{s})$, the high-gear equivalent viscous damping coefficient; $J_{\text {eq }}=2.087 \times 10^{-3} \mathrm{Kg}-\mathrm{m}^{2}$, high-gear equivalent moment of inertia without external load. Then, evaluate $J_{\text {eq }}$ assuming disk load is attached to the high gear. Next, theoretically evaluate $B_{\text {eq }, v}$ and $A_{\mathrm{m}}$. Performing the bump test in the laboratory, we find the value of open-loop gain, $K=1.53 \mathrm{rad} / \mathrm{s} / \mathrm{V}$, and by gain at cutoff frequency $=0.0254 \mathrm{~s}$.

Hence, the open-loop transfer function for position control application by the SRV02 is given by

$G(s)=\frac{1.53}{s(0.0254 s+1)}$.

QUBE-Servo-2 As per the discussion given in the user manual [34], the QUBE-Servo-2 has a brushed DC motor that is connected to a PWM amplifier. Encoders are used here to measure angular position. There are many types of encoders, but we have used here in this experiment the rotary incremental optical encoder, and the angle they measure depends on the last position and when it was last powered. The Quanser is a direct-drive rotary servo system as shown in Fig. 4. As the encoder resolution is 512 lines per revolution and goes up to 2048 in quadrature mode, to get a measurement in degrees we need a gain of $360^{\circ} / 2048$ cnts $=0.1758^{\circ} / \mathrm{cnts}$.

The motor armature circuit schematic of QUBE-Servo-2 is shown in Fig. 10, and the electrical and mechanical parameters are terminal resistance, $R_{\mathrm{m}}=8.4 \Omega$, torque constant, $K_{\mathrm{t}}=0.042 \mathrm{~N} \mathrm{~m} / \mathrm{A}$, motor back-emf constant, $K_{\mathrm{m}}=0.042 \mathrm{~V} /(\mathrm{rad} / \mathrm{s})$, rotor inertia, $J_{\mathrm{m}}=4.0 \times 10^{6} \mathrm{~kg} \mathrm{~m}^{2}$, load hub inertia, $J_{\mathrm{h}}=0.6 \times 10^{6} \mathrm{~kg} \cdot \mathrm{m}^{2}$, load hub radius, $r_{\mathrm{h}}=0.0111 \mathrm{~m}$, load hub mass, $m_{\mathrm{h}}=0.0106 \mathrm{~kg}$, rotor inductance, $L_{\mathrm{m}}=1.16 \mathrm{~m} \mathrm{H}$, mass of load disk, $M_{\mathrm{d}}=0.053 \mathrm{~kg}$, and radius of disk load, $r_{\mathrm{d}}=0.0248 \mathrm{~m}$. The DC motor shaft is connected to a load hub. The hub is a metal disk used to mount the disk or rotary pendulum and has a moment of inertia of $J_{\mathrm{h}}$. A disk load is attached to the output shaft with a moment of inertia of $J_{\mathrm{d}}$.

Here, in QUBE-Servo-2 the back-emf (electromotive) voltage $e_{b}(t)$ depends on the speed of the motor shaft, $w_{\mathrm{m}}$ and the back-emf constant of the motor, $k_{\mathrm{m}}$. It opposes the current flow. The electrical equation of this system is similar to the electrical equation of SRV02. The back-emf voltage is same as Eq. 12. Also, using Kirchhoff's voltage law, the equation is same as in Eq. 13 and the value of $I_{\mathrm{m}}(t)$ is similar to Eq. 14.

Next, the motor shaft equation is expressed as

$J_{\text {eq }} w_{\mathrm{m}}(t)=\tau_{\mathrm{m}}(t)$

where $J_{\text {eq }}$ is total moment of inertia of a disk about its pivot, with mass $m$ and radius $r$, and is given by

$J=1 / 2 m r^{2}$

Based on the model, we have designed a model that applies a voltage and reads the position using the encoder. Based on the parameters, we can calculate the total moment of inertia acting on the motor shaft:

$J_{\text {eq }}=J_{m}+J_{h}+J_{d}$

The moment of inertia of the hub and disk load is:

$J_{\mathrm{h}}=1 / 2 m_{\mathrm{h}} r_{\mathrm{h}}^{2}$

$J_{\mathrm{d}}=1 / 2 m_{\mathrm{d}} r_{\mathrm{d}}^{2}$

Therefore, $J_{\text {eq }}=2.09 \times 10^{-5}$

From the bump test modeling which is based on step response of a stable system, the resulting steady-state gain is: $K=22.7 \mathrm{rad} /(\mathrm{Vs})$ and the time constant is: $\tau=0.158 \mathrm{~s}$ and the voltage-to-speed transfer function of the identified model becomes
Fig. 4 QUBE-Servo 2 DC motor and load

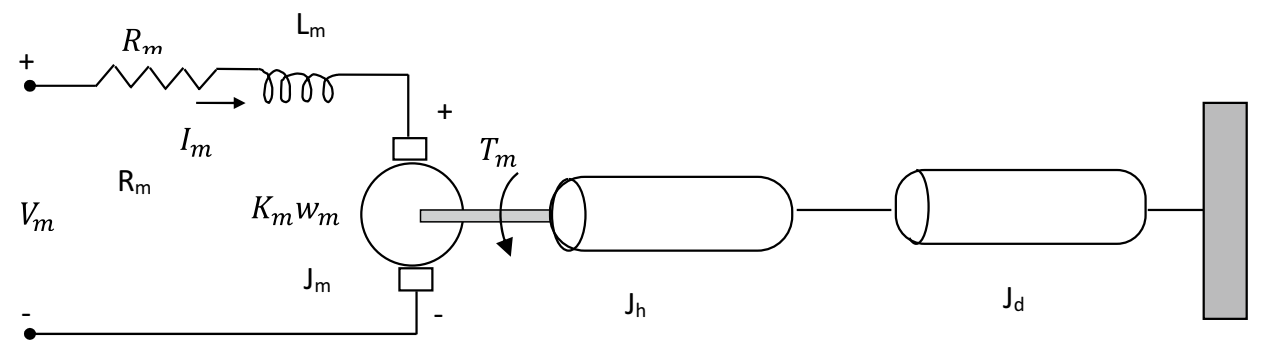

SN Applied Sciences 
$\frac{\Omega_{\mathrm{m}}(s)}{V_{\mathrm{m}}(s)}=\frac{22.7}{0.158 s+1}$

And the voltage-to-position transfer function is

$P(s)=\frac{\theta_{\mathrm{m}}(s)}{V_{\mathrm{m}}(s)}=\frac{22.7}{s(0.158 s+1)}$

\subsection{Simulation results}

As discussed in the above section from Eqs. 33 and 41, DC servo position control system may be given by first-order integrating plus delay (FOIPD) model:

$G_{p}(s)=\frac{K e^{-\theta_{d} s}}{s(\tau s+1)}$

Here, we consider time constant, $\tau=1 \mathrm{~s}$, dead time $\theta_{\mathrm{d}}=0.1 \mathrm{~s}$, and open-loop gain, $K=1$. Performance of IT2FLC is compared with GA-IT2-FLC, DE-IT2-FLC, PSO-IT2-FLC, CS-IT2-FLC, BCA-IT2-FLC, and CPSODE-IT2-FLC. The fitness value, execution time, and the values of $G_{e}, G_{\Delta e}, G_{\mathrm{Pl}}$, and $G_{\mathrm{PD}}$ after repeated runs are given in Table 3 . Robustness of the IT2-FLC is also evaluated for $50 \%$ increased value of dead time (i.e., $\theta_{d}=0.15 s$ ), along with the optimal
IT2-FLC. Responses for the nominal mode of the controller are given in Fig. 5. Performance indices for the nominal and perturbed models (with dead time $50 \%$ increased) are given in Table 6 . The performance indices are found to be improved for CS-IT2-FLC, DE-IT2-FLC, PSO-IT2-FLC,

Table 6 Comparison performances for model in (42)

\begin{tabular}{clllll}
\hline$\theta_{\mathrm{d}}$ & Controller & $\% \mathrm{OS}$ & $t_{\mathrm{s}}(s)$ & IAE & ITAE \\
\hline 0.1 & IT2-FLC & 20.1 & 46.8 & 14.64 & 531.9 \\
& CS-IT2-FLC & 1.4 & 17.1 & 7.268 & 151.5 \\
& DE-IT2-FLC & 00.7 & 13.9 & 3.518 & 29.98 \\
& GA-IT2-FLC & 1.9 & 18.8 & 7.842 & 180.6 \\
& PSO-IT2-FLC & 19.3 & 5.5 & 8.094 & 260.0 \\
& BCA-IT2-FLC & 0.2 & 10 & 3.303 & 48.48 \\
& CPSODE-IT2-FLC & 1.62 & 19.1 & 11.69 & 300.7 \\
0.15 & IT2-FLC & 20.2 & 46.3 & 14.70 & 535.2 \\
& CS-IT2-FLC & 1.5 & 19.2 & 7.275 & 152 \\
& DE-IT2-FLC & 0.03 & 21.2 & 3.513 & 34.36 \\
& GA-IT2-FLC & 1.8 & 15.00 & 6.587 & 168.4 \\
& PSO-IT2-FLC & 1.4 & 23.4 & 8.397 & 279.6 \\
& BCA-IT2-FLC & 0.02 & 14.00 & 3.41 & 55.92 \\
& CPSODE-IT2-FLC & 2.7 & 29.2 & 11.67 & 299.7 \\
\hline
\end{tabular}
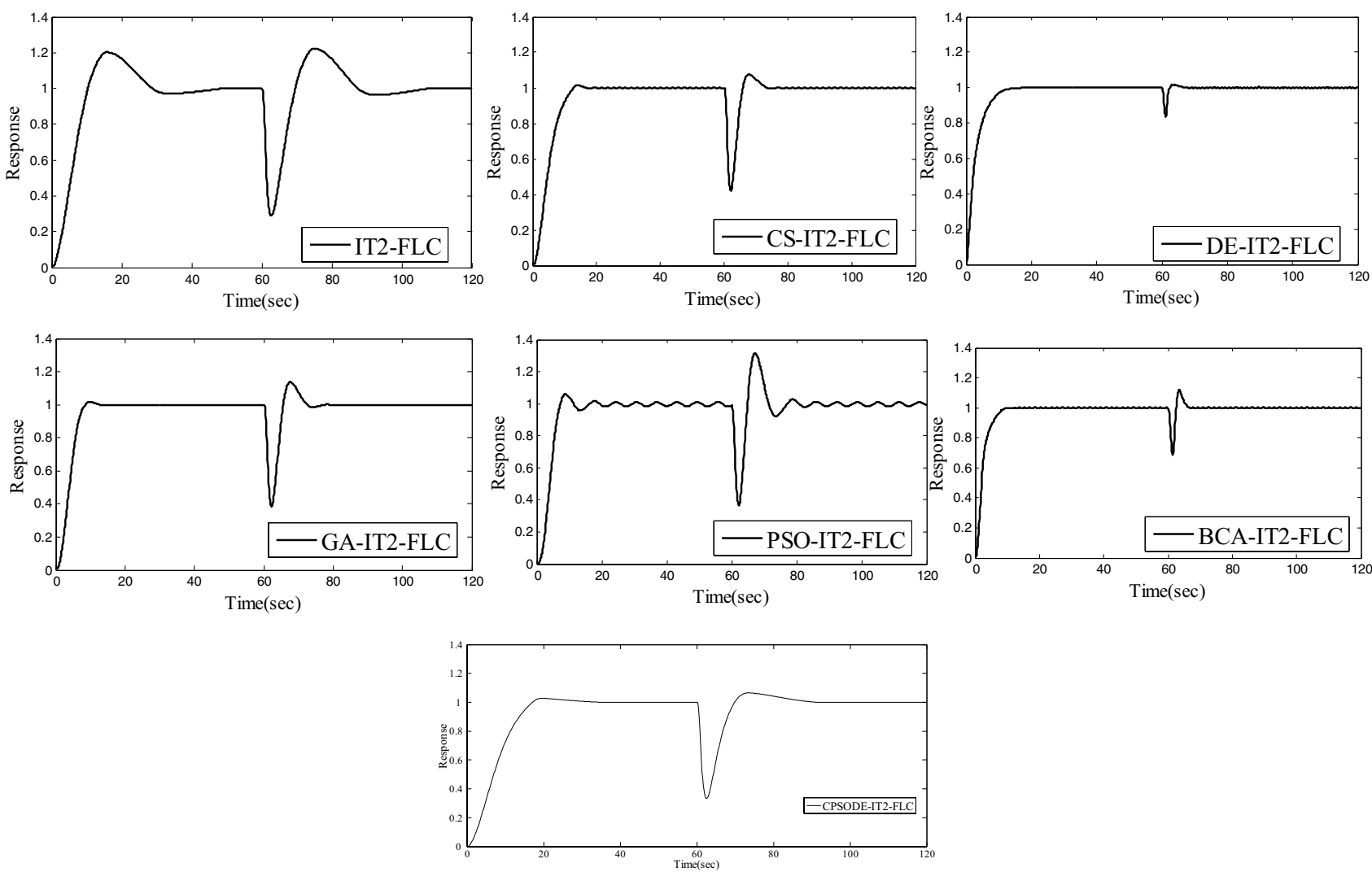

Fig. 5 Responses of IT2-FLC using different optimizations for model in (42) with dead time 0.15 

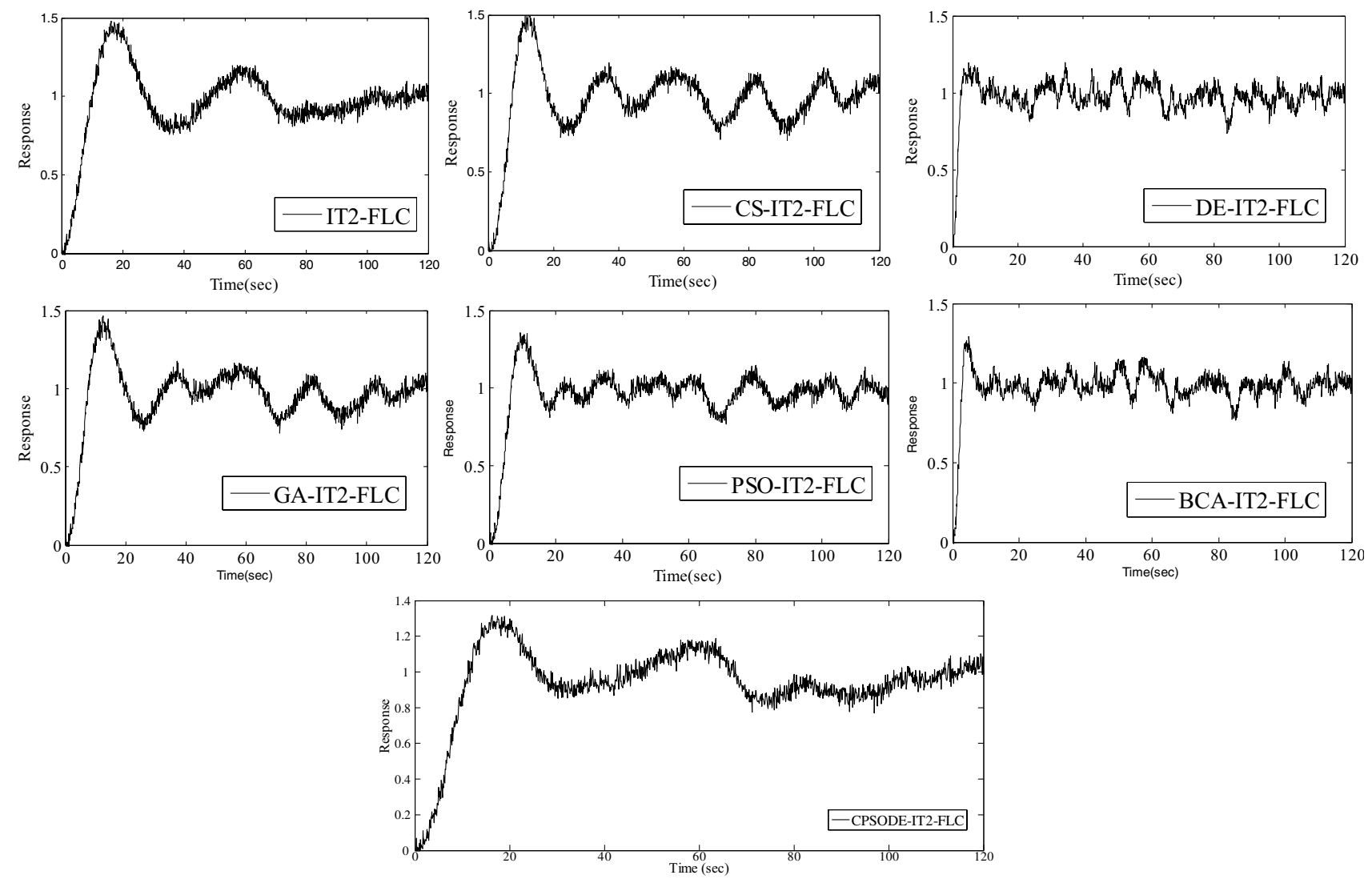

Fig. 6 Responses of IT2-FLC using different optimizations for model in (42) with noise

Table 7 Performance analysis for model in (42) with noise power 0.02

\begin{tabular}{llcl}
\hline$\theta_{\mathrm{d}}$ & Controller & IAE & ITAE \\
\hline 0.1 & IT2-FLC & 18.82 & 625.6 \\
& CS-IT2-FLC & 17.92 & 688.9 \\
& DE-IT2-FLC & 8.687 & 413.4 \\
& GA-IT2-FLC & 16.12 & 598.5 \\
& PSO-IT2-FLC & 11.89 & 424.1 \\
& BCA-IT2-FLC & 8.404 & 374.4 \\
& CPSODE-IT2-FLC & 17.11 & 527.1 \\
& IT2-FLC & 16.99 & 534 \\
& CS-IT2-FLC & 13.59 & 536.8 \\
& DE-IT2-FLC & 8.66 & 418.5 \\
& GA-IT2-FLC & 16.19 & 600.3 \\
& PSO-IT2-FLC & 12.07 & 434.1 \\
& BCA-IT2-FLC & 8.503 & 378.8 \\
& CPSODE-IT2-FLC & 17.28 & 539.1 \\
\hline
\end{tabular}

GA-IT2-FLC, BCA-IT2-FLC, and CPSODE-IT2-FLC compared to IT2-FLC. Similarly, the responses with noise power 0.02 are depicted in Fig. 6 and performance indices in Table 7.
DE-IT2-FLC performance is found to be superior compared to GA, BCA, PSO and BCA, and CPSODE-optimized IT2-FLC (Tables 6 and 7).

\subsection{Experimental results}

Efficiency of the IT2-FLC and optimized IT2-FLC is experimentally verified on Quanser, Canada SRV02 and QUBEServo-2 position control system. The snapshot of actual experimental setup of Quanser SRV02 is given in Fig. 7 and QUBE-Servo-2 in Fig. 8. For both the systems, the desired angular position (set value) is given through PC. Quanser SRV02 and QUBE-Servo-2 position control are identified in Sect. 4.2 as first-order integrating plus time delay (FOIPD) model and derived as

for SRV02, $\quad G_{\mathrm{SRVO} 2}(s)=\frac{1.53}{s(0.0254 s+1)}$.

and QUBE-Servo-2, $\quad G_{\text {QUBE }}(s)=\frac{22.7}{s(0.158 s+1)}$. 


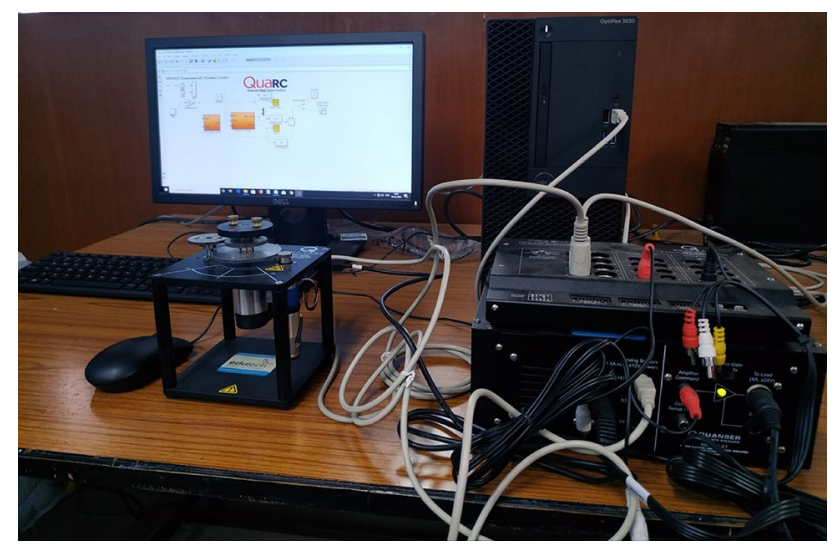

Fig. 7 Experimental setup of SRV02

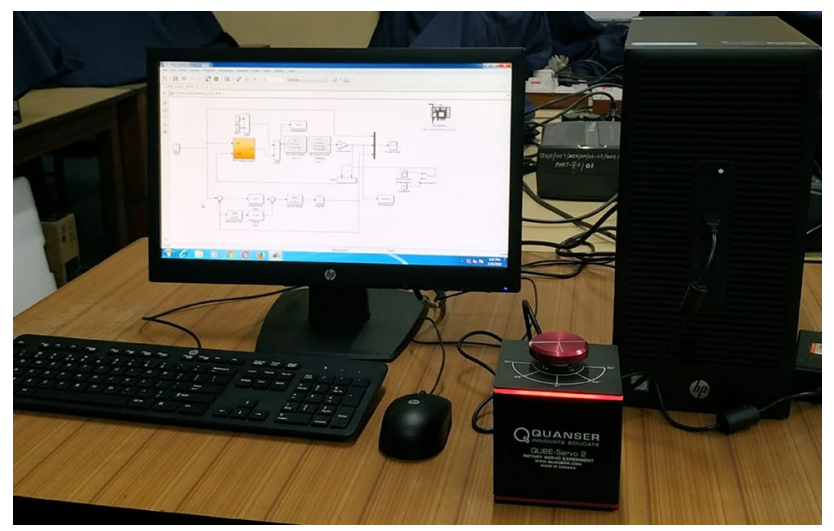

Fig. 8 Experimental setup of QUBE-Servo 2

With the QUARC and MATLAB-Simulink, reported controllers are implemented and their performances are evaluated. Performance study of set-point tracking and load regulation with IT2-FLC, CS-IT2-FLC, DE-IT2-FLC, PSOIT2-FLC, GA-IT2-FLC, BCA-IT2-FLC, and CPSODE-IT2-FLC is shown in Fig. 9 for SRV02 and Fig. 10 for QUBE-Servo-2. The comparative study for the controllers is depicted in Tables 8 and 9.

Performance of SRV02 and QUBE-Servo- 2 is also studied in the presence of measurement noise for IT2-FLC and with optimal IT2-FLC (Figs. 9, 10, 11, 12). Here, noise power applied for SRV02 with sample time 0.002 is $10^{-6}$, and similarly, noise power 0.02 for QUBE-Servo-2 with sample time is 0.01 . The related responses with measurement noise are given in Fig. 11 for SRV02 and Fig. 12 for QUBE-Servo-2, and analysis of performance indices is given in Tables 10 and 11.

Here based on the response-related performance indices, it is clear that applying optimization algorithms in determining the value of $G_{\mathrm{e}^{\prime}} G_{\Delta e^{\prime}} G_{\mathrm{P},}$ and $G_{\mathrm{PD}}$ of the IT2$\mathrm{FLC}$ there is immense improvement in close-loop response under model uncertainty and in the presence of measurement noise. Moreover, comparative study shows performance applying CPSODE optimization algorithm in Type-2 fuzzy controllers for practical systems is more superior to all the other heuristic algorithms GA, PSO, CS, DE, and BCA.

\section{Conclusion}

This paper present a comparative study of six types of nature-inspired optimization algorithms: the cuckoo search (CS) algorithm, the differential evolution (DE) algorithm, the particle swarm optimization (PSO), the genetic algorithm (GA), the bee colony algorithm (BCA), and the combined PSO and DE algorithm (CPSODE) to perform the input and output scaling parameters of the interval Type-2 fuzzy PID controller for servo systems. The comparison performances are in terms of the performance indices of systems responses under set-point tracking, load disturbances, and measurement noise. The analysis of simulation demonstrates a high performance of the differential evolution (DE) algorithm producing low percentage overshoot (\% O.S.), IAE, and ITAE for simulation. The experimental result with two servo systems SRV02 and QUBE-Servo-2 depicts CPSODE algorithm has superior performance despite the fact that the execution time for computation of the optimal parameter values is high compared to other algorithms. 

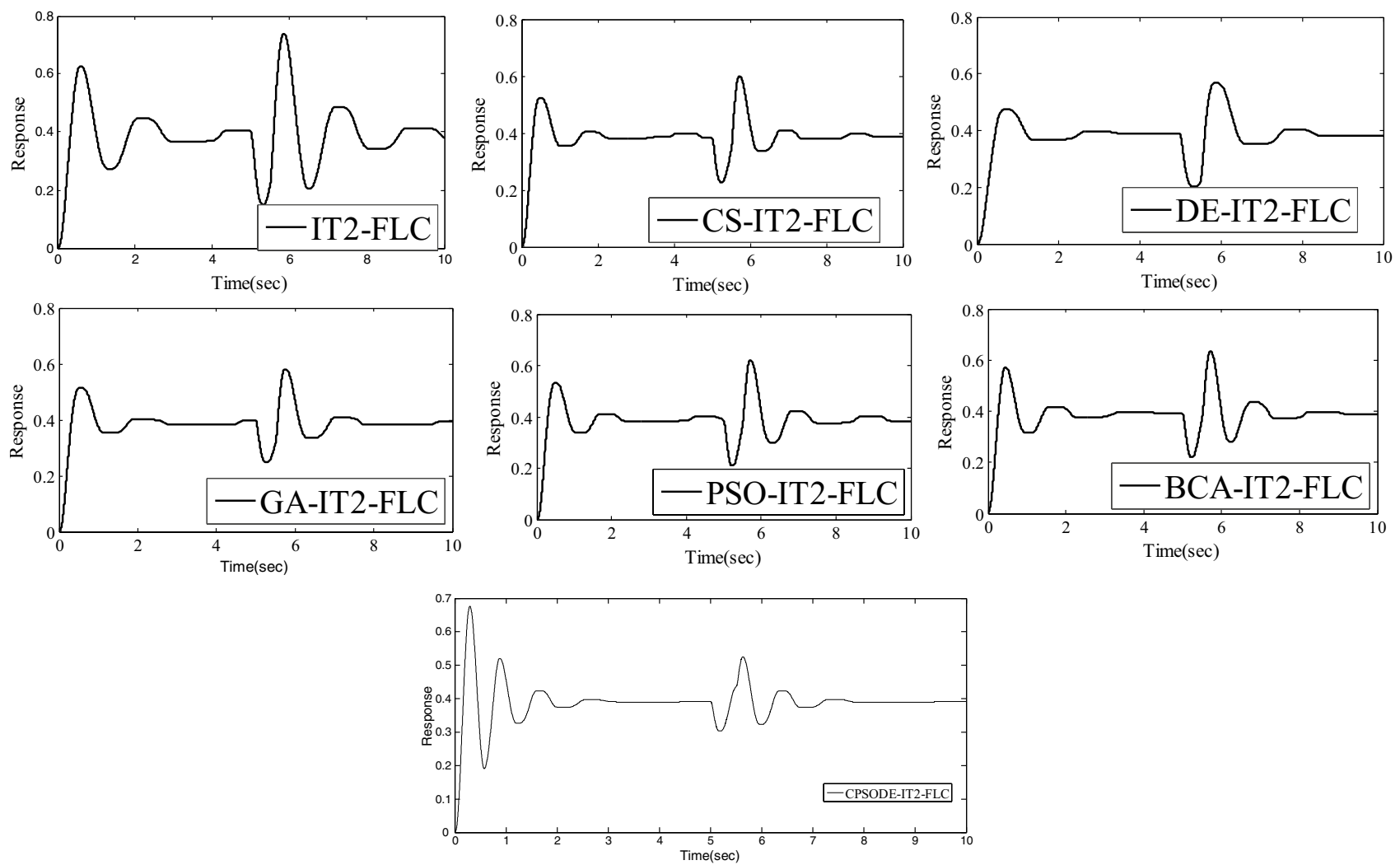

Fig. 9 Responses of IT2-FLC using different optimizations on SRV02
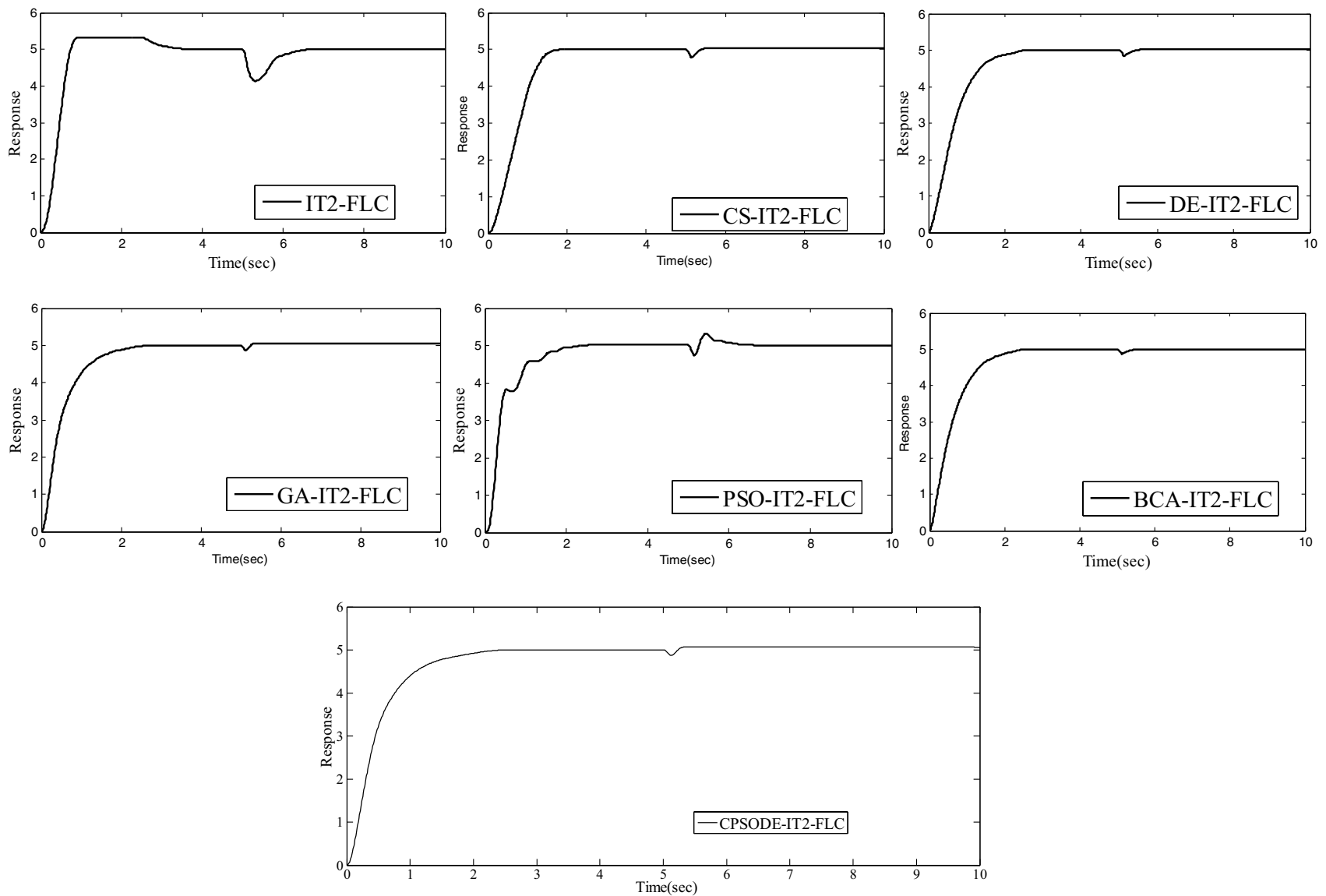

Fig. 10 Responses of IT2-FLC using different optimizations on Servo-2 QUBE 
Table 8 Comparison performances for SRV02 process

\begin{tabular}{lrlll}
\hline Controller & $\%$ OS & $t_{\mathrm{s}}(s)$ & IAE & ITAE \\
\hline IT2-FLC & 23.59 & 4.416 & 0.7774 & 3.270 \\
CS-IT2-FLC & 13.00 & 2.380 & 0.3499 & 1.236 \\
DE-IT2-FLC & 8.40 & 2.732 & 0.4352 & 1.625 \\
GA-IT2-FLC & 12.15 & 2.732 & 0.3479 & 1.200 \\
PSO-IT2-FLC & 14.21 & 2.414 & 0.4073 & 1.537 \\
BCA-IT2-FLC & 18.02 & 3.282 & 0.4067 & 1.481 \\
CPSODE-IT2-FLC & 28.23 & 2.698 & 0.3000 & 0.7387 \\
\hline
\end{tabular}

Table 9 Comparison performances for QUBE-Servo-2

\begin{tabular}{lllll}
\hline Controller & $\% O S$ & $t_{s}(s)$ & IAE & ITAE \\
\hline IT2-FLC & 6.7 & 3.62 & 0.6847 & 1.017 \\
CS-IT2-FLC & 0.2 & 1.84 & 0.7553 & 0.4804 \\
DE-IT2-FLC & 0.0 & 2.56 & 0.7086 & 0.5053 \\
GA-IT2-FLC & 0.0 & 2.79 & 0.6145 & 0.6623 \\
PSO-IT2-FLC & 1.4 & 2.52 & 0.5413 & 0.4703 \\
BCA-IT2-FLC & 0.8 & 2.73 & 0.6419 & 0.3827 \\
CPSODE-IT2-FLC & 0.1 & 2.39 & 0.5776 & 0.7306 \\
\hline
\end{tabular}
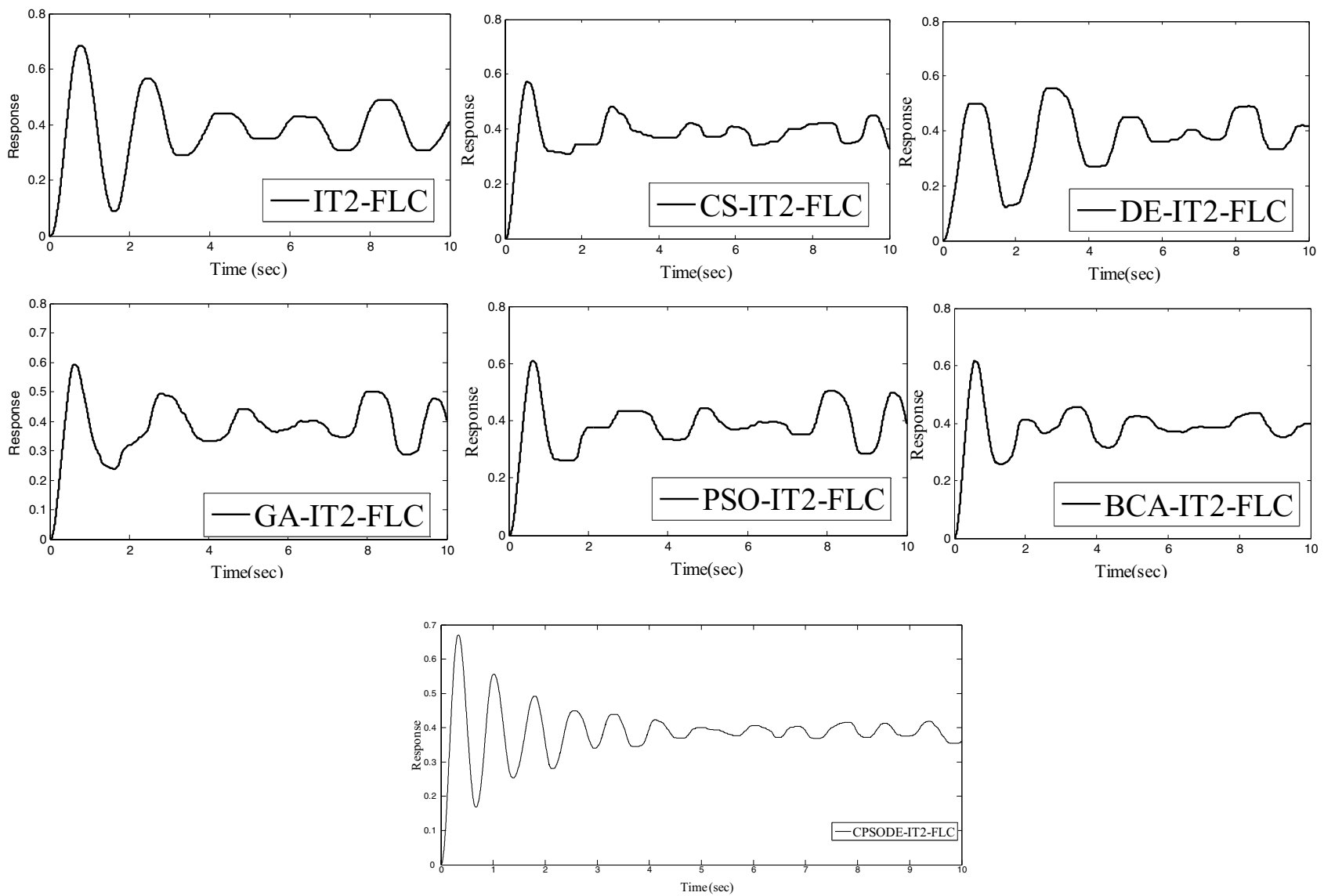

Fig. 11 Responses of IT2-FLC using different optimizations on SRV02 with noise (noise factor $10^{-6}$ ) 

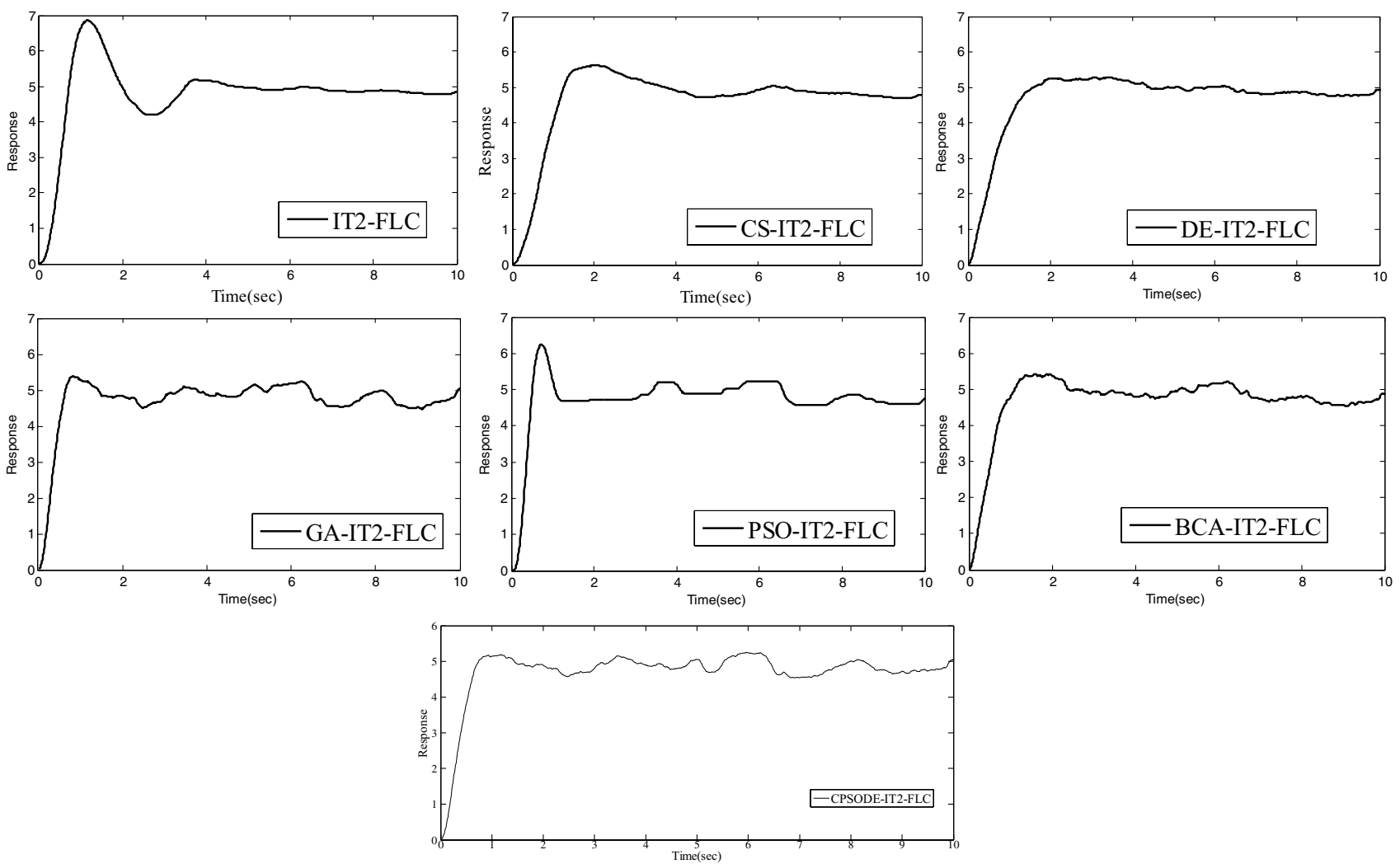

Fig. 12 Responses of IT2-FLC using different optimizations on Servo-2 QUBE with noise (noise factor 0.02)

Table 10 Performance analysis for SRV02 process with noise

\begin{tabular}{lll}
\hline Controller & IAE & ITAE \\
\hline IT2-FLC & 0.9280 & 3.208 \\
CS-IT2-FLC & 0.4915 & 1.732 \\
DE-IT2-FLC & 0.8924 & 3.062 \\
GA-IT2-FLC & 0.6955 & 2.835 \\
PSO-IT2-FLC & 0.6456 & 2.739 \\
BCA-IT2-FLC & 0.5166 & 1.646 \\
CPSODE-IT2-FLC & 0.4848 & 1.4333 \\
\hline
\end{tabular}

\section{Compliance with ethical standards}

Conflict of interest On behalf of all authors, the corresponding author states that there is no conflict of interest.

\section{References}

1. Ali Al-Waily RS (2010) Design of robust mixed $\mathrm{H} 2 / \mathrm{H} \infty$ PID controller using particle swarm optimization. Int J Adv Comput Technol 2(5):53-60

2. Krohling RA, Rey JP (2001) Design of optimal disturbance rejection PID controllers using genetic algorithm. IEEE Trans Evolut Comput 5(1):78-82
Table 11 Performance analysis for QUBE-Servo-2 with noise

\begin{tabular}{lll}
\hline Controller & IAE & ITAE \\
\hline IT2-FLC & 1.074 & 2.139 \\
CS-IT2-FLC & 1.101 & 2.290 \\
DE-IT2-FLC & 0.899 & 1.849 \\
GA-IT2-FLC & 0.780 & 2.597 \\
PSO-IT2-FLC & 0.869 & 2.742 \\
BCA-IT2-FLC & 0.862 & 2.509 \\
CPSODE-IT2-FLC & 0.7311 & 2.251
\end{tabular}

3. Castillo O, Amador-Angulo L (2017) A generalized type-2 fuzzy logic approach for dynamic parameter adaptation in bee colony optimization applied to fuzzy controller design. Inf Sci 460-461:476-496

4. Dell'Orco M, Marinelli M, Altieri MG (2017) Solving the gate assignment problem through the fuzzy bee colony optimization. Trans Res C Emerg Technol 80:424-438

5. Konar M, Bagis A (2016) Performance comparison of particle swarm optimization, differential evolution and artificial bee colony algorithms for fuzzy modelling of nonlinear systems. Elektron Elektrotech 22:8-13

6. Holland J (1975) Adaptation in natural and artificial systems. The University of Michigan Press, AnnArbor

7. Haupt RL, Haupt SE (2004) Practical genetic algorithms. Wiley, New York 
8. Kennedy J, Eberhart RC (1995) Particle swarm optimization. In: Proceedings of IEEE international conference on neural networks, Piscataway, NJ, pp 1942-1948

9. Storn R, Price KV (1997) Differential evolution—a simple and efficient heuristic for global optimization over continuous spaces. J Global Optim 11:341-359

10. Karaboga D, Basturk B (2008) On the performance of artificial bee colony $(A B C)$ algorithm. Appl Soft Comput 8:689-697

11. Yang XS, Deb S (2009) Cuckoo search ViaLevy flights. In: Nature \& biologically inspired computing, 2009, World Congress on (IEEE 2009), pp 210-214

12. Zhang L, Fu Q, Chen Jio, Bai H, Zhou X (2017) A modified particle swarm optimization algorithm-CPSODE. In: 29th Chinese control and decision conference (CCDC). IEEE, pp 6659-6663

13. Hagras HA (2004) A hierarchical type-2 fuzzy logic control architecture for autonomous mobile robots. IEEE Trans Fuzzy Syst 12(4):524-539

14. Hassani H, Zarei J (2015) Interval type-2 fuzzy logic controller design for speed control of DC motors. Syst Sci Control Eng $3: 266-273$

15. Zadeh LA (1975) The concept of a linguistic variable and its application to approximate reasoning-1. Inf Sci 8:199-249

16. Karnik NN, Mendel JM (1998) Introduction to type-2 fuzzy logic systems. Proc IEEE Int Conf Fuzzy Syst 2:915-920

17. Mendel JM (2001) Uncertain rule-based fuzzy logic systems: introduction and new directions. Prentice-Hall, New Jersey

18. Mendel JM (2007) Advances in type-2 fuzzy sets and systems. Inf Sci 177(1):84-110

19. Mendel J, John R (2002) Type-2 fuzzy sets made simple. IEEE Trans Fuzzy Syst 10:117-127

20. Karnik NN, Mendel JM (2001) Centroid of a type-2 fuzzy set. Inform Sci 132:195-220

21. Wu D, Tan WW (2006) A simplified Type-2 fuzzy logic controller for real-time control. ISA Trans 45(4):503-516

22. Ontiveros-Robles E, Melin P, Castillo O (2018) Comparative analysis of noise robustness of type-2 fuzzy logic controller. Kybernetika 54(1):175-201

23. Sakalli B, Kumbasar T (2017) On the design and gain analysis of IT2-FLC with a case study on an electric vehicle. IEEE Int Confer Fuzzy Syst 25(6):1752-1764
24. Kumbasar T (2016) Robust stability analysis and systematic design of single-input interval type-2 fuzzy logic controller. IEEE Trans Fuzzy Syst 24(3):675-694

25. Kumar A, Kumar V (2017) A novel interval type-2 fractional order fuzzy PID controller: design, performance evaluation and its optimal time domain tuning. ISA Trans 68:251-275

26. Türkay D, Baykasoglu A, Altun K, Durmusoglu A, Türksen B (2011) Industrial applications of type-2 fuzzy sets and systems: a concise review. Comput Ind 62(1):125-137

27. Oh S-K, Jang H-J, Pedrycz W (2011) A comparative experimental study of Type-1/Type-2 fuzzy cascade controller based on genetic algorithms and particle swarm optimization. Expert Syst Appl 38:11217-11229

28. Bhoyar DV, Chilke BJ, Kemekar SS (2016) Design and analysis of type-2 fuzzy PID controller using genetic algorithm. Int J Recent Innov Trends Comput Commun 4(5):135-138

29. Castillo O, Melin P (2014) A review on interval type-2 fuzzy logic applications in intelligent control. Inf Sci 279:615-631

30. Ei-Nagar AM, Ei-Bardini M (2017) Parallel realization of self-tuning interval type-2 fuzzy controller. Eng Appl Artif Intell 61:8-20

31. Qiao WZ, Mizumoto M (1996) PID type fuzzy controller and parameters adaptive method. Fuzzy Sets Syst 78(1):23-35

32. Yesil E, Kumbasar T, Dodurka F, and Sakalli A (2014) Peak observer based self-tuning type-2 fuzzy PID controllers, In: Proceedings of international conference on artificial intelligence applications and innovations AIAI, pp 487-497

33. (2016) Documentation for the USER MANUAL SRV02. Quanser, Ontario, Canada

34. (2016) Documentation for the USER MANUAL QUBE-Servo-2, Quanser, Ontario, Canada

35. Palm R (1992) Sliding mode fuzzy control. In: Proceedings of IEEE International Conference on Fuzzy Systems-FUZZ-IEEE, pp 519-526

Publisher's Note Springer Nature remains neutral with regard to jurisdictional claims in published maps and institutional affiliations. 